\title{
Influence of Omalizumab on Allergen-Specific IgE in Patients with Adult Asthma
}

\author{
Hiroko Mizuma ${ }^{a}$ Akihiko Tanaka ${ }^{a}$ Yoshitaka Uchida $^{a}$ Akiko Fujiwara ${ }^{a}$ \\ Ryo Manabe $^{a}$ Hitomi Furukawa $^{a}$ Naota Kuwahara $^{a}$ Yosuke Fukuda ${ }^{a}$ \\ Tomoyuki Kimura $^{a}$ Megumi Jinno $^{a}$ Shin Ohta ${ }^{a}$ Mayumi Yamamoto ${ }^{a}$ \\ Satoshi Matsukarac ${ }^{c}$ Mitsuru Adachi ${ }^{\mathrm{b}}$ Hironori Sagara ${ }^{a}$
}

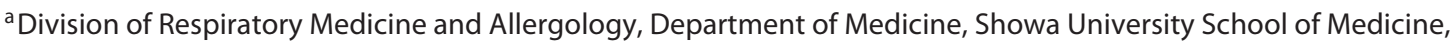
and ${ }^{b}$ Department of Allergy, Sanno Hospital, Clinical Research Centers for Medicine, International University of Health and Welfare, Tokyo, and ' Department of Respiratory Medicine, Showa University Fujigaoka Hospital,

Yokohama, Japan

\section{Key Words}

Omalizumab · Anti-immunoglobulin E · Allergic asthma ·

Seroconversion

\begin{abstract}
Background: Omalizumab, an anti-immunoglobulin E (lgE) monoclonal antibody, inhibits the binding of circulating lgE to mast cells and basophils, resulting in fewer episodes of airway inflammation, asthma symptoms and exacerbations in patients with severe allergic asthma. Treatment of patients with asthma using omalizumab increases serum total $\lg \mathrm{E}(\mathrm{t} \lg \mathrm{E})$ levels. However, little is known about the influence of omalizumab on allergen-specific lgE (slgE). Methods: tlgE and slgE in 47 adult patients with severe asthma were measured with a fluorescent enzyme immunoassay (Immuno(AP-FEIA) before and after omalizumab treatment. Results: Treatment with omalizumab increased tlgE and slgE levels. The increases in slgE by class category after omalizumab treatment were positively correlated with baseline slgE positivity before treatment. The mean changes in slgE levels after omalizumab treatment were also correlated with base-
\end{abstract}

(C) 2016 S. Karger AG, Basel

$1018-2438 / 16 / 1683-0165 \$ 39.50 / 0$ line slgE levels before treatment. The mean changes in tlgE levels were positively correlated with the mean changes in IgE levels against Dermatophagoides pteronyssinus, crude house dust, Japanese cedar and moth. Omalizumab markedly influenced the negative-to-positive seroconversion rate for IgE against Japanese cedar (30.8\%), Candida (29.0\%) and moth (28.0\%). Finally, all patients with negative-to-positive seroconversion for Japanese cedar-specific lgE had cedar pollinosis before beginning omalizumab treatment. Conclusions: The changes in slgE levels after omalizumab treatment may be dependent on the baseline slgE levels. Our data may indicate the presence of undetectable but functional slgE.

๑ 2016 S. Karger AG, Basel

\section{Introduction}

Immunoglobulin E (IgE), discovered by Ishizaka [1] and Johansson [2] in 1966, plays a key role in allergic reactions. Levels of total $\operatorname{IgE}(\mathrm{t} \operatorname{IgE})$ and allergen-specific $\operatorname{IgE}$ (sIgE) are helpful for diagnosing allergic diseases such as

\section{KARGER 125}

E-Mail karger@karger.com www.karger.com/iaa
Correspondence to: Dr. Akihiko Tanaka

Division of Respiratory Medicine and Allergology, Department of Medicine Showa University, School of Medicine

1-5-8 Hatanodai, Shinagawa-ku, Tokyo, 142-8666 (Japan)

E-Mail tanakaa@med.showa-u.ac.jp 
asthma, allergic rhinitis and atopic dermatitis. IgE is a critical factor for the development of bronchial hyperresponsiveness in patients with asthma [3]. Several epidemiological studies have demonstrated that the tIgE level is higher in patients with asthma than in those without asthma [4-6]. In addition, the tIgE level in children with severe asthma is significantly higher than in children with mild to moderate asthma [7]. Global multicenter studies, such as SARP (Severe Asthma Research Program) and ENFUMOSA (European Network for Understanding Mechanisms of Severe Asthma), have demonstrated that there is no correlation between the tIgE level and asthma severity $[8,9]$. Serum tIgE levels peak during early adolescence and decrease with age [10-12]. However, longitudinal changes in IgE level are heterogeneous among adult patients with asthma. Recently, we found that longitudinal increases in tIgE levels are associated with poor disease control in patients with adult asthma [13]. However, the association between IIgE level and asthma control has not been fully elucidated. Moreover, the association between sIgE levels and asthma severity remains unknown.

Omalizumab, a humanized anti-human IgE monoclonal antibody, is an adjunct treatment option for severe persistent allergic IgE-mediated asthma in addition to the optimized standard therapy for patients with asthma aged $\geq 6$ years. The clinical effects of omalizumab on patients with severe asthma revealed that IgE-mediated mechanisms, which cannot be suppressed by steroids or leukotriene receptor antagonists, play an important role in severe asthma. Omalizumab reduces free IgE levels by approximately $95 \%$ by binding to the ce 3 region on free $\operatorname{IgE}$, thereby blocking the binding of free IgE to its specific, high-affinity receptor on mast cells, basophils and any other cells expressing IgE receptors. Omalizumab does not interact with membrane-bound $\operatorname{IgE}$. By reducing $\operatorname{IgE}$ levels, omalizumab leads to the downregulation of FceRI on mast cells and basophils [14]. Omalizumab has a long half-life (19-22 days), in part due to its slow removal by the hepatic reticuloendothelial system [15]. The half-life of omalizumab-bound IgE is longer than that of free $\operatorname{IgE}$ [16], and clinical tests cannot discriminate between free IgE and omalizumab-bound IgE. Therefore, administration of omalizumab generally increases serum IgE levels in patients with asthma [17]. Several reports have measured free IgE levels separately from omalizumab-bound $\operatorname{IgE}[18,19]$, but the studies concerned did not involve a clinical setting.

Although omalizumab is known to increase clinically detectable tIgE levels, changes in sIgE levels $(\Delta \operatorname{sIgE})$ have not been investigated thus far. Therefore, we observed $\Delta$ sIgE after omalizumab treatment and the influence of omalizumab treatment on these changes.

\section{Materials and Methods}

\section{Study Participants}

Eighty-nine patients with severe adult asthma were treated with omalizumab for at least 4 months in the Showa University Hospital, Japan, from April 2009 to March 2014. After excluding 42 patients, owing to lack of data regarding levels of tIgE and/or sIgE, 47 were included in the study. Thirty patients with asthma who were not receiving omalizumab were included in the control group. The data on tIgE and sIgE levels were retrospectively collected. Asthma was diagnosed according to the criteria of the Global Strategy for Asthma Management and Prevention by the Global Initiative for Asthma (GINA) guideline [20]. Japanese cedar pollinosis was diagnosed according to clinical symptoms and the presence of Japanese cedar-specific IgE. Patients with chronic obstructive pulmonary disease or other lung diseases, a smoking history of $>20$ pack-years, vocal-cord dysfunction or neurological disease were excluded. Diagnoses for perennial allergic rhinitis and seasonal cedar pollinosis were based on a clinical history and positive serum sIgE test results. The baseline data included demographic details (age, sex and basal BMI), clinical features (age at onset), smoking status (ex-smoker, current smoker or never-smoker), Asthma Control Test score, spirometry and fractional exhaled nitric oxide (FeNO). The study protocol was reviewed and approved by the Showa University Ethics Committee, and written informed consent was obtained from each subject.

\section{Study Design}

Omalizumab was administered subcutaneously at 2- or 4 -week intervals depending on the patient's body weight and tIgE level at screening. Blood samples were obtained before and after omalizumab treatment. tIgE levels ranged from 30 to 700 $\mathrm{IU} / \mathrm{ml}$. The clinical effectiveness of omalizumab was assessed by means of the Global Evaluation of Treatment Effectiveness (GETE) by the physician at 16 weeks $[21,22]$. The GETE has 5 categories: (1) complete control of asthma, (2) a marked improvement of asthma, (3) a discernible but limited improvement in asthma, (4) no appreciable change in asthma and (5) worsening of asthma. We used the first 2 levels of the GETE to define a treatment response.

\section{Data Collection}

Blood was collected from the patients at 2 time points: before beginning treatment with omalizumab, and shortly before the omalizumab injection at least 16 weeks or a maximum of 30 weeks after treatment with omalizumab. The blood collection period among control patients ranged from 20 to 40 weeks. The serum tIgE and sIgE levels were measured with a fluorescent enzyme immunoassay (ImmunoCAP-FEIA, Phadia AB, Uppsala, Sweden). sIgE levels were determined for 11 aeroallergens: cocksfoot, ragweed, Cryptomeria japonica (Japanese cedar), Dermatophagoides pteronyssinus (Der p), crude house dust, Aspergillus fumigatus (aspergillus), Candida albicans (candida), Alternaria alternate (alter-
Mizuma et al. 
Table 1. Patient demographics

\begin{tabular}{|c|c|c|c|}
\hline & $\begin{array}{l}\text { Controls } \\
(\mathrm{n}=30)\end{array}$ & $\begin{array}{l}\text { Omalizumab group } \\
(\mathrm{n}=47)\end{array}$ & $\mathrm{p}$ value \\
\hline Age, years & $57.5 \pm 12.6$ & $58.1 \pm 17.6$ & 0.612 \\
\hline Gender M:F (of M) & $11: 19(36.7)$ & $12: 35(25.5)$ & 0.298 \\
\hline BMI & $23.6 \pm 3.9$ & $22.5 \pm 3.2$ & 0.219 \\
\hline Age at onset of asthma, years & $35.9 \pm 20.2$ & $35.8 \pm 18.8$ & 0.726 \\
\hline Smoking history current:ex-smoker:never & $0: 6: 24$ & $0: 10: 37$ & 0.893 \\
\hline Pet ownership & $6(20.0)$ & $13(27.7)$ & 0.447 \\
\hline Allergic rhinitis & $17(56.7)$ & $24(51.0)$ & 0.765 \\
\hline Cedar pollinosis & $19(63.3)$ & $29(61.7)$ & 0.886 \\
\hline Asthma Control Test & $22.4 \pm 2.67$ & $14.2 \pm 3.92$ & $<0.001$ \\
\hline Peripheral eosinophils, / $\mu$ l & $378.7 \pm 429.7$ & $362.1 \pm 498.4$ & 0.477 \\
\hline $\mathrm{tIgE}, \mathrm{U} / \mathrm{l}$ & $432.0 \pm 456.1$ & $424.0 \pm 451.0$ & 0.774 \\
\hline$\%$ FVC, \% & $92.5 \pm 16.9$ & $78.5 \pm 22.6$ & 0.006 \\
\hline$\% \mathrm{FEV}_{1}, \%$ & $83.9 \pm 16.9$ & $67.4 \pm 24.7$ & 0.002 \\
\hline $\mathrm{FEV}_{1.0 \%}, \%$ & $74.7 \pm 11.2$ & $74.6 \pm 17.7$ & 0.762 \\
\hline FeNO, ppb & $57.0 \pm 37.6$ & $46.3 \pm 35.7$ & 0.076 \\
\hline
\end{tabular}

naria), cat dander, dog dander and moth. sIgE levels were classified: $<0.35 \mathrm{kU} / \mathrm{l}$ (class 0), 0.35-0.69 kU/l (class 1), 0.70-3.49 kU/1 (class 2), 3.50-17.49 kU/1 (class 3), 17.50-49.99 kU/1 (class 4), $50.00-100.00 \mathrm{kU} / \mathrm{l}$ (class 5) and $>100.00 \mathrm{kU} / \mathrm{l}$ (class 6). The changes in $\operatorname{tgE}(\Delta \mathrm{tIgE})$ or $\Delta$ sIgE were calculated as follows: tIgE after omalizumab - tIgE before omalizumab or sIgE after omalizum$\mathrm{ab}-$ sIgE before omalizumab.

However, $\triangle$ sIgE were assessed only in the participants whose sIgE levels were $>0.35 \mathrm{kU} / \mathrm{l}$ after omalizumab treatment. Sensitization was defined as an sIgE level $\geq 0.35 \mathrm{kU} / \mathrm{l}$.

Spirometry was performed using an AS-302 spirometer (Minato Medical Science Co. Ltd., Osaka, Japan) in accordance with American Thoracic Society/European Respiratory Society guidelines [23] to determine $\mathrm{FEV}_{1}$, forced vital capacity (FVC) and $\mathrm{FEV}_{1} / \mathrm{FVC}\left(\mathrm{FEV}_{1 \%}\right)$. FeNO was measured using a portable device $\left(\right.$ NIOX MINO ${ }^{\circledR}$, Aerocrine AB, Solna, Sweden) at an expiratory flow rate of $50 \mathrm{ml} / \mathrm{s}$ for $10 \mathrm{~s}$.

\section{Statistical Analysis}

The results are expressed as mean \pm standard deviation or standard error of the mean for continuous variables. Statistical analyses were performed using JMP v10 (SAS Institute Inc., Cary, N.C., USA). The Pearson's correlation coefficient ( $r$ ) and Spearman rank order correlation coefficient $(\mathrm{R})$ were used to measure the correlation between the sIgE positivity before omalizumab treatment and the percent increase in sIgE levels by class after treatment, the correlation between the baseline levels of sIgE before treatment and the mean $\Delta \operatorname{sigE}$ and the correlation between the mean $\Delta \mathrm{tIgE}$ and the mean $\Delta$ sIgE. The differences in the continuous variables were analyzed using the Wilcoxon rank-sum test or the Kruskal-Wallis test, and the differences in the categorical variables were analyzed using the Pearson $\chi^{2}$ test. A $p$ value $<0.05$ was considered significant for all statistical assessments.

Influence of Omalizumab on sIgE

\section{Results}

\section{Study Participants}

The patient demographics are shown in table 1. All participants in the omalizumab group underwent treatment step 4 and above, according to the GINA guidelines before and after omalizumab treatment. In the control group, 20 patients underwent treatment step 3, 7 underwent treatment step 4 and 3 underwent treatment step 5. There were significant differences in the Asthma Control Test score, $\% \mathrm{FVC}$ and $\% \mathrm{FEV}_{1}$ between the control group and the omalizumab group. No difference was observed in peripheral eosinophils and tIgE levels. No patient was diagnosed with perennial conjunctivitis. Twenty-six patients (55.3\%) were classified as displaying 'complete control of asthma' or 'a marked improvement of asthma' at 16 weeks of omalizumab treatment, thus indicating the omalizumab responders. Ten patients owned dogs, 2 owned cats and 1 owned a bird.

\section{$\Delta$ sIgE after Omalizumab Treatment}

The prevalence of sIgE is shown in figure 1. Sensitization was defined as an sIgE level $\geq 0.35 \mathrm{kU} / \mathrm{l}$. Der $\mathrm{p}$-specific IgE (76.6\%), crude house dust-specific IgE (76.6\%) and cedar-specific IgE (71.7\%) showed high positivity. $\Delta \mathrm{tIgE}$ and $\Delta \mathrm{sIgE}$ after omalizumab treatment are shown in table 2. There were significant differences in $\Delta \mathrm{tIgE}$ and $\Delta$ sIgE except for $\Delta$ alternaria-specific IgE between the 


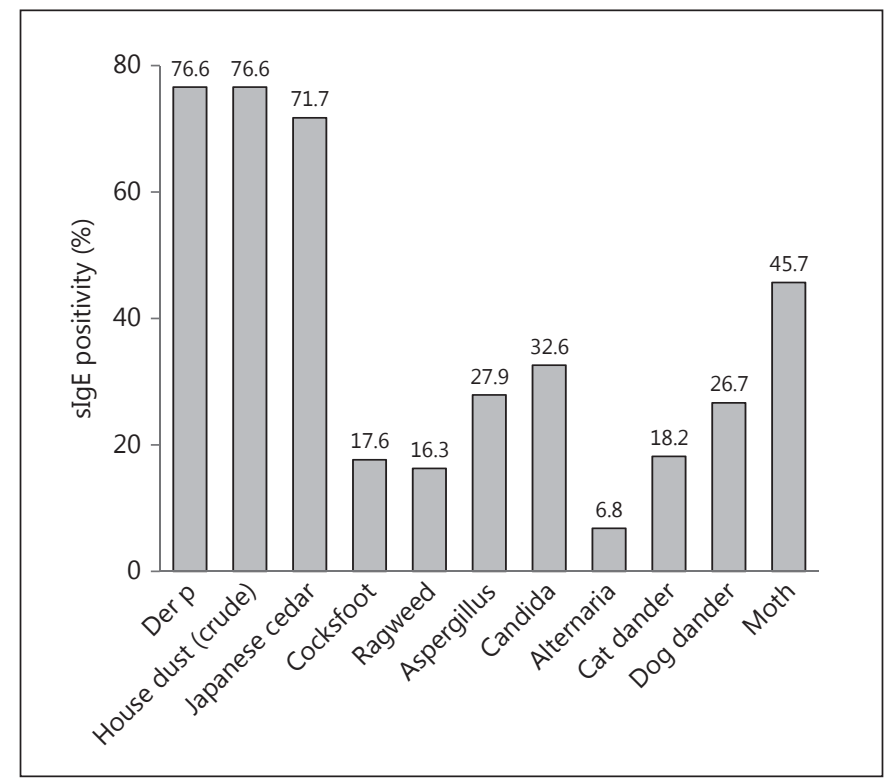

Fig. 1. sIgE positivity in patients before omalizumab treatment was measured using ImmunoCAP-FEIA $(\mathrm{n}=47)$. Sensitization was defined as an sIgE level $>0.35 \mathrm{kU} / \mathrm{l}$. Values are shown above the bars.

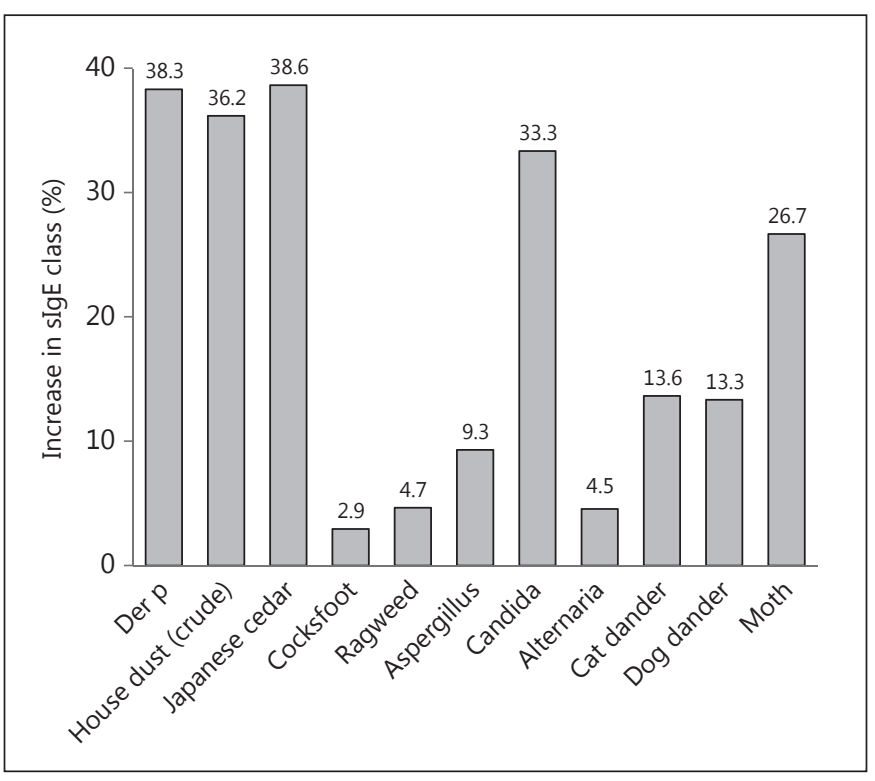

Fig. 2. Percent increase in sIgE levels by class category after omalizumab treatment $(n=47)$. Values are shown above the bars.

control group and the omalizumab group. sIgE levels for alternaria were $<0.35 \mathrm{kU} / \mathrm{l}$ in $41 / 44$ patients before omalizumab treatment. The raw data for tIgE and sIgE before and after omalizumab treatment are shown in online supplementary table S1 (for all online suppl. material, see
Table 2. Mean changes in tIgE and sIgE after omalizumab

\begin{tabular}{lclr}
\hline & $\begin{array}{l}\text { Controls } \\
(\mathrm{n}=30)\end{array}$ & $\begin{array}{l}\text { Omalizumab } \\
\text { group } \\
(\mathrm{n}=47)\end{array}$ & $\mathrm{p}$ value \\
& $-67.1 \pm 307.5$ & $326.6 \pm 372.1$ & $<0.001$ \\
\hline tIgE & $-0.4 \pm 4.5$ & $6.6 \pm 11.8$ & $<0.001$ \\
$\Delta \mathrm{sIgE}$ & $-0.1 \pm 4.8$ & $5.2 \pm 11.9$ & $<0.001$ \\
Der p & $-2.8 \pm 13.7$ & $9.0 \pm 17.6$ & $<0.001$ \\
House dust (crude) & $-0.1 \pm 1.9$ & $1.5 \pm 4.8$ & 0.003 \\
Japanese cedar & $-0.1 \pm 0.4$ & $0.3 \pm 1.0$ & 0.002 \\
Cocksfoot & $0.1 \pm 0.3$ & $0.5 \pm 0.8$ & 0.046 \\
Ragweed & $-0.02 \pm 0.2$ & $0.4 \pm 0.6$ & $<0.001$ \\
Aspergillus & $0.01 \pm 0.1$ & $0.4 \pm 1.8$ & 0.485 \\
Candida & $-0.5 \pm 2.0$ & $1.0 \pm 3.0$ & 0.005 \\
Alternaria & $-0.4 \pm 2.3$ & $0.3 \pm 0.9$ & 0.011 \\
Cat dander & $-0.1 \pm 0.6$ & $0.9 \pm 2.6$ & $<0.001$ \\
Dog dander & & & \\
Moth & &
\end{tabular}

Values $(\mathrm{kU} / \mathrm{l})$ express changes as means $\pm \mathrm{SD}$.

www.karger.com/doi/10.1159/000442668). As the durations of omalizumab treatment varied widely, we determined the differences in $\Delta \mathrm{tIgE}$ and $\Delta \mathrm{sIgE}$ between the early time point group (at 16-22 weeks) and the late time point group (at 23-30 weeks). No difference was observed in $\Delta$ tIgE and $\Delta$ sIgE for Der $p$, crude house dust, Japanese cedar, aspergillus, candida, dog and moth between the early time point group and the late time point group (online suppl. table S2). The percent increase in sIgE levels by $\geq 1$ classes after omalizumab treatment is shown in figure 2. The percent increase in Der p-specific IgE (38.3\%), crude house dust-specific IgE (36.2\%), cedar-specific IgE (38.6\%), candida-specific IgE (33.3\%) and moth-specific $\operatorname{IgE}(26.7 \%)$ was considerably high.

\section{Correlation between sIgE before Omalizumab and $\triangle$ sIgE after Omalizumab}

We investigated the correlations between $\operatorname{sIgE}$ before omalizumab treatment and $\Delta$ sIgE. Figure 3 a shows a positive correlation between sIgE positivity before omalizumab treatment and the percent increase in $\operatorname{sIgE}$ by class after treatment $(\mathrm{R}=0.833, \mathrm{p}=0.001)$. A positive correlation was also observed between baseline sIgE levels before omalizumab treatment and $\Delta \operatorname{sgE}$ (fig. $3 \mathrm{~b} ; \mathrm{R}=0.825, \mathrm{p}=$ $0.001)$. In addition, there was no significant difference in the percent increase between each individual sIgE (online suppl. fig. S1). Neither $\Delta \mathrm{tg} E$ nor $\Delta s \operatorname{IgE}$ were associated with the cumulative doses of omalizumab (data not shown). 


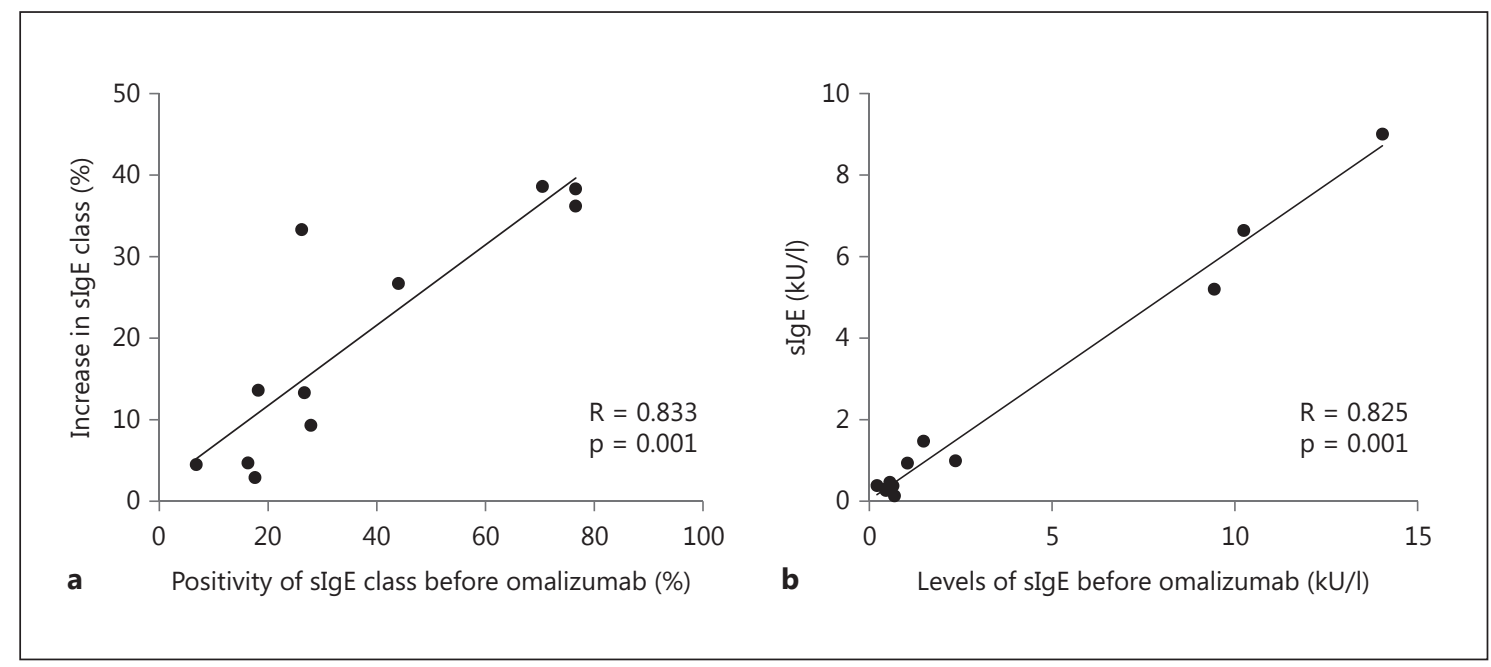

Fig. 3. a Correlation between sIgE positivity before omalizumab treatment and the percent increase in sIgE levels by class category after omalizumab treatment. b The correlation between sIgE levels before omalizumab treatment and the mean change in sIgE levels after omalizumab treatment.

Next, we investigated the correlation between $\Delta \mathrm{tg} \mathrm{E}$ and $\Delta \mathrm{sIgE}$. Analyses were performed in patients with detectable sIgE levels $(>0.35 \mathrm{kU} / \mathrm{l})$ after omalizumab treatment. A positive correlation was observed between $\Delta \mathrm{tIgE}$ and mean changes in IgE levels against Der $\mathrm{p}$, crude house dust, cedar and moth (table 3). However, no correlation was detected between $\Delta \mathrm{tIgE}$ and mean changes in IgE levels against aspergillus, candida and dog dander (table 3 ), despite the high correlation coefficient between $\Delta \mathrm{t} \operatorname{IgE}$ and mean changes in aspergillusIgE.

\section{Negative-to-Positive Seroconversion in sIgE}

Finally, we investigated the negative-to-positive seroconversion rate for sIgE, which was class 0 at baseline, after omalizumab treatment. The number of samples in class 0 at baseline was 11 for Der p, 11 for HD, 13 for Japanese cedar, 28 for cocksfoot, 36 for ragweed, 31 for aspergillus, 31 for candida, 41 for alternaria, 36 for cat dander, 33 for dog dander and 25 for moth. Highly marked negative-to-positive seroconversion rates for IgE against Japanese cedar (30.8\%), candida (29.0\%) and moth (28.0\%) were observed (fig. 4). Intriguingly, all patients with negative-to-positive seroconversion for Japanese cedar-specific IgE had cedar pollinosis even before beginning omalizumab treatment. The blood was collected in the pollen season from $1 / 4$ patients who showed cedar-specific IgE seroconversion after omalizumab treatment.

Influence of Omalizumab on sIgE
Table 3. Correlation between $\Delta \mathrm{tIgE}$ and $\Delta \mathrm{sIgE}$

\begin{tabular}{lllrl}
\hline & $\begin{array}{l}\text { Total } \\
\text { samples, } \\
\mathrm{n}\end{array}$ & $\begin{array}{l}\text { Valid } \\
\text { samples, } \\
\mathrm{n}\end{array}$ & $\mathrm{R}$ value & $\mathrm{p}$ value \\
& & 36 & 0.496 & 0.002 \\
\hline tIgE vs. $\Delta$ Der p-IgE & 47 & 36 & 0.485 & 0.003 \\
$\Delta$ tIgE vs. $\Delta$ HD-IgE & 47 & 36 & 0.479 & 0.005 \\
$\Delta$ tIgE vs. $\Delta$ cedar-IgE & 44 & 35 & 0.484 & 0.094 \\
$\Delta$ tIgE vs. $\Delta$ Asp-IgE & 43 & 13 & 0.116 & 0.608 \\
$\Delta$ tIgE vs. $\Delta$ candida-IgE & 42 & 23 & 0.266 & 0.404 \\
$\Delta$ tIgE vs. $\Delta$ dog-IgE & 45 & 14 & 0.516 & 0.012 \\
$\Delta$ tIgE vs. $\Delta$ moth-IgE & 45 & 27 & & \\
\hline
\end{tabular}

a Samples in which $\operatorname{sigE}$ was at a detectable level $(\geq 0.35 \mathrm{kU} / \mathrm{l})$ before and after or at least after treatment with omalizumab. Asp = aspergillus; $\mathrm{HD}=$ house dust (crude).

\section{Discussion}

This study examined $\Delta \mathrm{sIgE}$ in patients with adult asthma after omalizumab treatment. We found that $\Delta$ sIgE after omalizumab were positively correlated with the level of sIgE before omalizumab treatment but not the allergen specificity. To the best of our knowledge, this study is the first to elucidate the influence of omalizumab on sIgE in patients with severe adult asthma.

We found positive correlations between $\Delta \mathrm{tIgE}$ and mean changes in the IgE levels against Der $\mathrm{p}$, crude house 


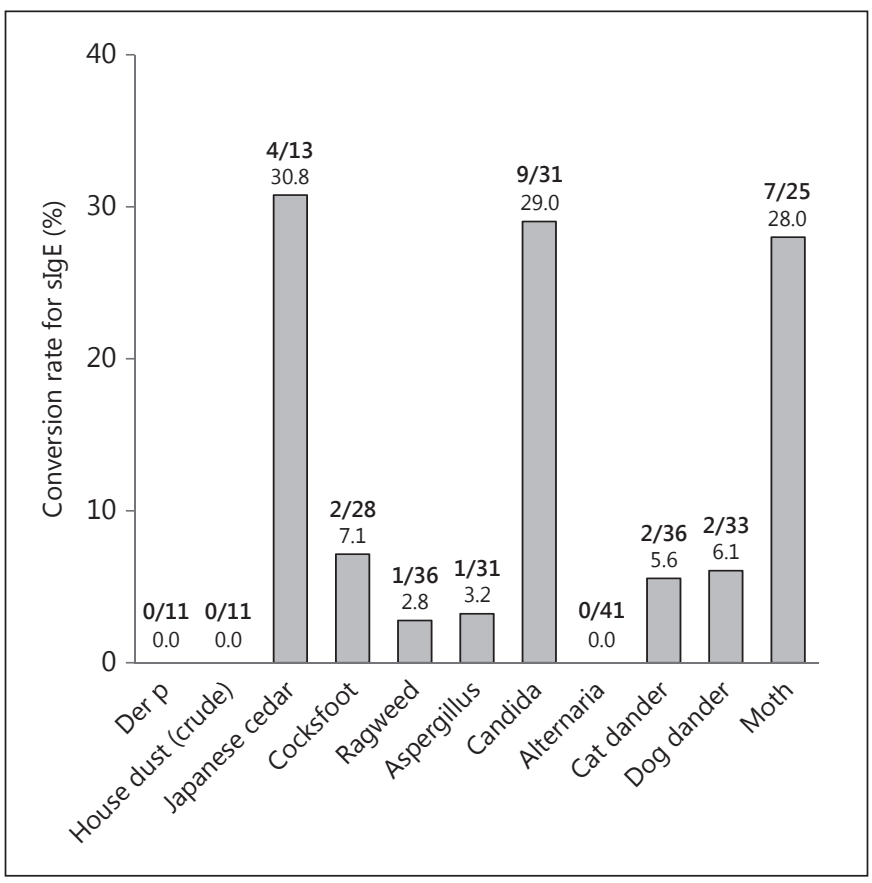

Fig. 4. Percentage of negative-to-positive seroconversion rates for sIgE. Raw data are shown above the bars. Numbers of patients appear in bold type.

dust, Japanese cedar and moth. Generally, the tIgE level reflects both specific and unspecific IgE. Previous birth cohort studies showed that the evolution of tIgE was extremely heterogeneous but parallel with that of airborne sIgE $[24,25]$, indicating that $\Delta \mathrm{tIgE}$ are dependent on antigen-specific IgE. Although both $\Delta \operatorname{tg} E$ and $\Delta$ sIgE were mostly influenced by omalizumab, our data are consistent with these previous data. However, there was no correlation between $\Delta \operatorname{tgEE}$ and the mean changes in the IgE levels against candida and dog dander. This suggests that $\Delta \mathrm{t} \operatorname{IgE}$ may be dependent on $\Delta \mathrm{sIgE}$, for which the baseline positivity was relatively high.

In this study, specific IgE levels against several allergens, which were $<0.35 \mathrm{kU} / \mathrm{l}$ before omalizumab treatment, increased to $>0.35 \mathrm{kU} / \mathrm{l}$, and these patients showed negative-to-positive seroconversion after omalizumab treatment. In particular, negative-to-positive seroconversion was frequently observed for IgE against Japanese cedar, candida and moth. IgE positivity for these specific allergens was considerably high before omalizumab treatment. Intriguingly, although Der p-specific and house dust-specific IgE showed a high positivity, similar to Japanese cedar-specific IgE, no negative-to-positive seroconversion was observed. Further studies with a large sample size are necessary to clarify these findings. Interestingly, all 4 patients with negative-to-positive seroconversion for Japanese cedar-specific IgE after omalizumab treatment were diagnosed with cedar pollinosis before the treatment, indicating the presence of functional sIgE, despite the level being maintained at $<0.35 \mathrm{IU} / \mathrm{l}$. However, we assume that seroconverted sIgEs do not have clinical significance in patients treated with omalizumab because they are not free to bind to mast cells and basophils.

Recently, omalizumab was found to be effective in treating patients with severe nonatopic asthma who did not respond to a skin-prick test and/or with negative $\operatorname{sigE}$ results [26-28]. One reason for the effectiveness of omalizumab in patients with nonatopic asthma may be the presence of local sIgE. In fact, Mouthuy et al. [29] reported that both tIgE and Der p-specific IgE in sputum were significantly increased in patients with nonatopic asthma compared to in healthy nonatopic participants. Our results indicate that the presence of undetectable sIgE due to the extremely low levels could be another reason for the effectiveness of omalizumab in patients with nonatopic asthma.

Currently, levels of specific IgE $>0.11 \mathrm{kU} / \mathrm{l}$ can be detected using ImmunoCAP-FEIA in a clinical setting in Japan. However, patients who began omalizumab treatment before 2011 showed levels of specific $\operatorname{IgE} \geq 0.35$ $\mathrm{kU} / \mathrm{l}$. Therefore, sensitization was defined as a specific IgE level $\geq 0.35 \mathrm{kU} / 1$. Extremely low specific IgE levels in combination with the methods used to measure these levels could be important. ImmunoCAP is the most common method used to measure tIgE and specific IgE levels. An alternative assay is the IMMULITE 2000 3gAllergy $^{\mathrm{TM}}$ (IMMULITE, Siemens Healthcare Diagnostics Inc., USA). IMMULITE employs liquid-phase kinetics in a bead format and a chemiluminescent enzyme immunoassay. The allergens are covalently attached to a soluble polymer matrix in IMMULITE and to a solid column in ImmunoCAP. The liquid-phase allergens increase the number of binding sites and their accessibility to the sIgE. In addition, IMMULITE has a wide measurement range, from 0.1 to $500 \mathrm{IUA} / \mathrm{ml}$ and a high degree of sensitivity [30-32]. Although the overall advantages of IMMULITE over ImmunoCAP have not been studied, IMMULITE seems to be a promising methodology with applicability in clinical settings. In this study, artificial increases in IgE by omalizumab shed light on the presence of clinically functional class 0 sIgE levels. Therefore, advances in the current methods used to measure high-sensitive IgE and re-examination of the class $0 \operatorname{IgE}$ level are necessary. 
This study had several limitations that need to be acknowledged. First, the time points of blood collection were not constant. The blood collections were performed from week 16 up to week 30 after beginning omalizumab treatment. Omalizumab treatment rapidly increases and then gradually decreases tIgE levels [33]. However, there is no previous report that describes the time-course of $\operatorname{tgE}$ and sIgE levels after omalizumab treatment. If samples were collected when the $\operatorname{IgE}$ level peaked, the $\operatorname{tg} \mathrm{E}$ and sIgE levels could have been higher. In addition, the frequency of negative-to-positive seroconversion for sIgE after omalizumab treatment could further increase. However, we demonstrated no difference in $\Delta$ sIgE between the early time point group and the late time point group. We therefore believe that even if blood was collected at the optimum time point, the positive correlation observed between $\Delta$ sIgE after omalizumab treatment and baseline levels of sIgE would not change. A previous study demonstrated that early $\Delta \mathrm{tIgE}$ can be used as a predictor of future responders to omalizumab [34]. In this study, only a single time point was used, i.e. after omalizumab treatment. Therefore, we could not assess the relationship between $\Delta$ sIgE and the major outcome variables of omalizumab, such as pulmonary function, symptom scores and oral corticosteroid use. Measurements of sIgE at different time points would provide further elucidation of the influence of omalizumab on sIgE. Second, this study involved a small sample size. Omalizumab is an expensive medicine, and opportunities to administer it are limited, even for patients with uncontrolled asthma. Therefore, it was relatively difficult to collect enough samples in a real-life setting. In particular, it was difficult to determine the frequency of negative-to-positive seroconversion for sIgE, because only those patients sensitized to perennial allergens are indicated for omalizumab treatment. Third, this study did not consider seasonal variations in IgE levels. The prevalence of cedar pollinosis in the study participants was $61.7 \%$. tIgE should increase in participants with cedar pollinosis in the Japanese cedar season, i.e. mostly in March and April, and just after this season. We showed that the percent increase in cedar-specific IgE, which was susceptible to seasonal variations, did not significantly differ from that observed for the other sIgEs. Thus, even though we standardized the season of omalizumab treatment and blood collection, we believe that the positive correlation observed between $\Delta \mathrm{sIgE}$ after omalizumab treatment and baseline levels of sIgE would not change.

In conclusion, we found that $\Delta s \operatorname{IgE}$ after omalizumab treatment were dependent on the baseline levels of sIgE. In addition, we also found that all patients with negativeto-positive seroconversion for cedar-specific IgE due to omalizumab treatment had cedar pollinosis before starting on omalizumab treatment. These findings suggest that sIgEs can play a critical role in allergic diseases despite being under the detectable level. Further larger-scale studies are needed to validate our data.

\section{Acknowledgments}

The authors thank Ms. Kyoko Inui and Ms. Manami Matsuda for their excellent assistance with the data collection and analysis.

\section{Disclosure Statement}

There were no potential conflicts of interest to declare.

\section{References}

1 Ishizaka K, Ishizaka T, Hornbrook MM: Physico-chemical properties of human reaginic antibody. IV. Presence of a unique immunoglobulin as a carrier of reaginic activity. J Immunol 1966;97:75-85.

2 Johansson SG, Bennich H: Immunological studies of an atypical (myeloma) immunoglobulin. Immunology 1967;13:381-394.

3 Rabe KF: Mechanisms of immune sensitization of human bronchus. Am J Respir Crit Care Med 1998;158:S161-S170.

4 Holford-Strevens V, Warren P, Wong C, Manfreda J: Serum total immunoglobulin E levels in Canadian adults. J Allergy Clin Immunol 1984;73:516-522.
5 Criqui MH, Seibles JA, Hamburger RN, Coughlin SS, Gabriel S: Epidemiology of immunoglobulin E levels in a defined population. Ann Allergy 1990;64:308-313.

6 Burrows B, Martinez FD, Halonen M, Barbee RA, Cline MG: Association of asthma with serum IgE levels and skin-test reactivity to allergens. N Engl J Med 1989;320:271-277.

7 Borish L, Chipps B, Deniz Y, Gujrathi S, Zheng B, Dolan CM, Group TS: Total serum IgE levels in a large cohort of patients with severe or difficult-to-treat asthma. Ann Allergy Asthma Immunol 2005;95:247-253.
8 Moore WC, Bleecker ER, Curran-Everett D, Erzurum SC, Ameredes BT, Bacharier L, Calhoun WJ, Castro M, Chung KF, Clark MP, Dweik RA, Fitzpatrick AM, Gaston B, Hew M, Hussain I, Jarjour NN, Israel E, Levy BD, Murphy JR, Peters SP, Teague WG, Meyers DA, Busse WW, Wenzel SE: Characterization of the severe asthma phenotype by the $\mathrm{Na}$ tional Heart, Lung, and Blood Institute's Severe Asthma Research Program. J Allergy Clin Immunol 2007;119:405-413.

9 The ENFUMOSA cross-sectional European multicentre study of the clinical phenotype of chronic severe asthma. European Network for Understanding Mechanisms of Severe Asthma. Eur Respir J 2003;22:470-477. 
10 Wittig HJ, Belloit J, De Fillippi I, Royal G: Age-related serum immunoglobulin E levels in healthy subjects and in patients with allergic disease. J Allergy Clin Immunol 1980;66: 305-313.

11 Grundbacher FJ, Massie FS: Levels of immunoglobulin $\mathrm{G}, \mathrm{M}, \mathrm{A}$, and $\mathrm{E}$ at various ages in allergic and nonallergic black and white individuals. J Allergy Clin Immunol 1985;75:651658.

12 Stoy PJ, Roitman-Johnson B, Walsh G, Gleich GJ, Mendell N, Yunis E, Blumenthal MN: Aging and serum immunoglobulin E levels, immediate skin tests, RAST. J Allergy Clin Immunol 1981;68:421-426.

13 Tanaka A, Jinno M, Hirai K, Miyata Y, Mizuma $\mathrm{H}$, Yamaguchi M, Ohta S, Watanabe $\mathrm{Y}$, Yamamoto M, Suzuki S, Yokoe T, Adachi M, Sagara H: Longitudinal increase in total IgE levels in patients with adult asthma: an association with poor asthma control. Respir Res 2014; 15:144.

14 Holgate S, Casale T, Wenzel S, Bousquet J, Deniz Y, Reisner C: The anti-inflammatory effects of omalizumab confirm the central role of IgE in allergic inflammation. J Allergy Clin Immunol 2005;115:459-465.

15 Hochhaus G, Brookman L, Fox H, Johnson C, Matthews J, Ren S, Deniz Y: Pharmacodynamics of omalizumab: implications for optimised dosing strategies and clinical efficacy in the treatment of allergic asthma. Curr Med Res Opin 2003;19:491-498.

16 Chang TW: The pharmacological basis of anti-IgE therapy. Nat Biotechnol 2000;18:157162.

17 Saini SS, MacGlashan DW, Sterbinsky SA, Togias A, Adelman DC, Lichtenstein LM, Bochner BS: Down-regulation of human basophil IgE and Fc epsilon RI alpha surface densities and mediator release by anti-IgEinfusions is reversible in vitro and in vivo. J Immunol 1999;162:5624-5630.

18 Ito R, Gon Y, Nunomura S, Atsuta R, Harada N, Hattori T, Maruoka S, Okayama Y, Ra C, Hashimoto S: Development of assay for determining free IgE levels in serum from patients treated with omalizumab. Allergol Int 2014; 63(suppl 1):37-47.
19 Korn S, Haasler I, Fliedner F, Becher G, Strohner P, Staatz A, Taube C, Buhl R: Monitoring free serum IgE in severe asthma patients treated with omalizumab. Respir Med 2012;106:1494-1500.

20 Global Initiative for Asthma (GINA): Global strategy for asthma management and prevention (updated report 2015). http://www.ginasthma.org/local/uploads/files/GINA_Report_2015.pdf (published April 2015).

21 Bousquet J, Siergiejko Z, Swiebocka E, Humbert M, Rabe KF, Smith N, Leo J, Peckitt C, Maykut R, Peachey G: Persistency of response to omalizumab therapy in severe allergic (IgEmediated) asthma. Allergy 2011;66:671-678.

22 Brusselle G, Michils A, Louis R, Dupont L, Van de Maele B, Delobbe A, Pilette C, Lee CS, Gurdain S, Vancayzeele S, Lecomte P, Hermans $\mathrm{C}$, MacDonald K, Song M, Abraham I: 'Real-life' effectiveness of omalizumab in patients with severe persistent allergic asthma: the PERSIST study. Respir Med 2009;103: 1633-1642.

23 Miller MR, Hankinson J, Brusasco V, Burgos F, Casaburi R, Coates A, Crapo R, Enright P, van der Grinten CP, Gustafsson P, Jensen R, Johnson DC, MacIntyre N, McKay R, Navajas $D$, Pedersen OF, Pellegrino R, Viegi G, Wanger J, Force AET: Standardisation of spirometry. Eur Respir J 2005;26:319-338.

24 Matricardi PM, Bockelbrink A, Grüber C, Keil T, Hamelmann E, Wahn U, Lau S: Longitudinal trends of total and allergen-specific IgE throughout childhood. Allergy 2009;64: 1093-1098.

25 Matricardi PM, Bockelbrink A, Keil T, Grüber C, Niggemann B, Hamelmann E, Wahn U, Lau S: Dynamic evolution of serum immunoglobulin E to airborne allergens throughout childhood: results from the Multi-Centre Allergy Study birth cohort. Clin Exp Allergy 2009;39:1551-1557.

26 de Llano LP, Vennera MeC, Álvarez FJ, Medina JF, Borderías L, Pellicer C, González H, Gullón JA, Martínez-Moragón E, Sabadell C, Zamarro S, Picado C, Registry S: Effects of omalizumab in non-atopic asthma: results from a Spanish multicenter registry. J Asthma 2013;50:296-301.
27 Menzella F, Piro R, Facciolongo N, Castagnetti C, Simonazzi A, Zucchi L: Long-term benefits of omalizumab in a patient with severe non-allergic asthma. Allergy Asthma Clin Immunol 2011;7:9.

28 van den Berge M, Pauw RG, de Monchy JG, van Minnen CA, Postma DS, Kerstjens HA: Beneficial effects of treatment with anti-IgE antibodies (omalizumab) in a patient with severe asthma and negative skin-prick test results. Chest 2011;139:190-193.

29 Mouthuy J, Detry B, Sohy C, Pirson F, Pilette C: Presence in sputum of functional dust mite-specific IgE antibodies in intrinsic asthma. Am J Respir Crit Care Med 2011;184: 206-214

30 Bernstein DI, Biagini RE, Karnani R, Hamilton R, Murphy K, Bernstein C, Arif SA, Berendts B, Yeang HY: In vivo sensitization to purified Hevea brasiliensis proteins in health care workers sensitized to natural rubber latex. J Allergy Clin Immunol 2003;111:610616.

31 Biagini RE, MacKenzie BA, Sammons DL, Smith JP, Krieg EF, Robertson SA, Hamilton RG: Latex specific IgE: performance characteristics of the IMMULITE 2000 3gAllergy assay compared with skin testing. Ann Allergy Asthma Immunol 2006;97:196-202.

32 Szecsi PB, Stender S: Comparison of immunoglobulin E measurements on IMMULITE and ImmunoCAP in samples consisting of allergen-specific mouse-human chimeric monoclonal antibodies towards allergen extracts and four recombinant allergens. Int Arch Allergy Immunol 2013;162:131-134.

33 Lowe PJ, Tannenbaum S, Gautier A, Jimenez P: Relationship between omalizumab pharmacokinetics, IgE pharmacodynamics and symptoms in patients with severe persistent allergic (IgE-mediated) asthma. Br J Clin Pharmacol 2009;68:61-76.

34 Dal Negro RW, Guerriero M, Micheletto C, Tognella S, Visconti M: Changes in total IgE plasma concentration measured at the third month during anti-IgE treatment predict future exacerbation rates in difficult-to-treat atopic asthma: a pilot study. J Asthma 2011; 48:437-441. 


\section{Erratum}

In the article by Mizuma et al., entitled 'Influence of omalizumab on allergen-specific IgE in patients with adult asthma' [Int Arch Allergy Immunol 2015;168:165-172, DOI: $10.1159 / 000442668]$, the third last author's name was misspelt and should read Matsukura. 